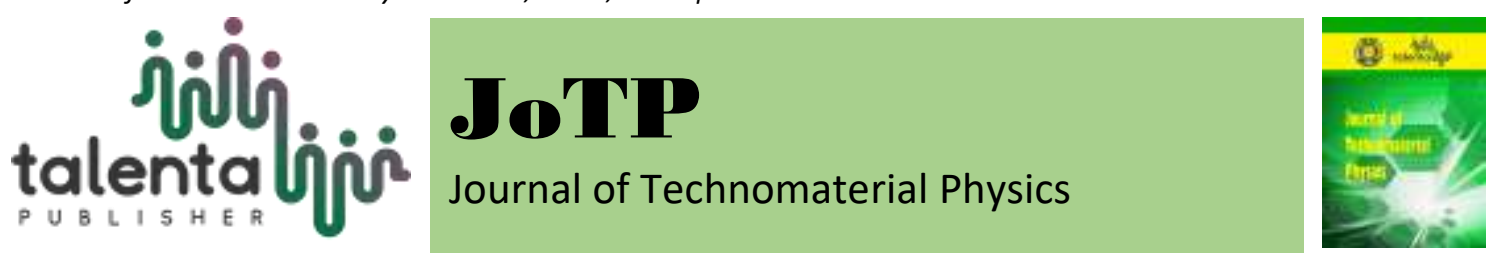

\title{
The Analysis of Hydrophobic Interaction on Aspergillus Niger Xylanase Enzyme Thermal Stability
}

\author{
Nia Daniaty Malau ${ }^{1}$ and Manogari Sianturi ${ }^{2}$ \\ ${ }^{1,2}$ Physics Education Program, Faculty of Teacher Training and Education, Universitas Kristen 13630 \\ Indonesia
}

\begin{abstract}
Xylanase is a type of enzyme that has an important role in the industrial field. One measure that can be done to improve the thermostability of an enzyme is by protein engineering. The mutation of the protein can be done by studying protein structures through molecular dynamics simulation approach. In this research, thermal stability analysis on Aspergilus niger Wild Type Xylase (AnX) was performed which aims to study the thermal stability characteristics of xynalase enzyme from Aspergillus niger through molecular dynamics simulation approach. AnX molecular dynamics simulation was performed by NAMD (Not Just Another Molecular Dynamic) software at 300 - 500 K. The research was focused on the study of enzyme thermal stability characteristics in order to get the information of residues accountable for such characteristics. The selection of residues to be mutated was based on hydrophocic interaction analysis. Then from that, the design of xylanase enzyme mutant with better thermostability than wild type xynalase enzyme was made in order to provide design reference for more stable xylanase mutation design which can be implemented in wet experiments for of Aspergillus niger Xylanase enzyme genetic engineering. The enzyme was unfolded at $500 \mathrm{~K}$ at $9.5 \mathrm{~ns}$. The residues responsible of the thermal stability were based on hydrophobic interaction analysis in Alanin at residue 60. This residue is located in segmen/chain 3. The best mutant is Alanin 60 residue mutant which is replaced by Methionin and $\Delta \Delta$ Gsolv of -21.10345 was obtained. Thus, Ala60Met is the most stable mutant which might increase the thermal stability of Aspergillus niger Xylanase Enzyme.
\end{abstract}

Keyword: enzyme, thermostability, mutation, molecular dynamics

Received 11 April 2019 | Revised [30 June 2019] | Accepted [31 August 2019]

\section{Introduction}

These days, the demand of enzyme usage in industrial field has increased rapidly and obtained an important position in industrial fields [1-3]. Enzyme is being used widely in both food and non-food sectors. Usually enzymes are used in bread, cheese and meat businesses while in nonfood industries, they are used in paper and detergent industries for instance. Xylanase is one of the enzymes which is widely used. The enzyme is able to hydrolyzed xylan [4-6]. Xylanase enzyme is often used in pre-bleaching chemical pulp industries [7-9]. In pulp bleaching process, xylanase acts as a facilitator to remove lignin-carbohydrate complex which was formed during

*Corresponding author at: Jl. Mayjen Sutoyo No. 2 Jakarta Timur, 13630, Indonesia

E-mail address: malaunyadaniaty@gmail.com 
the pulping process. Therefore the enzyme easily reacts with bleacher and increase lignin extraction [10]. Xylanase used is able to decrease chlorine compounds content by $20-40 \%$ and improve paper quality obtained [11] and [10]. Besides that, the application of xylanase enzyme in pulp bleaching process is able to press down the cost compared to oxygen delignification, extended cooking and ozone substitution processes. Xylanase characteristics which are suitable for industrial use in bleaching are high temperature resistance, which is around $60-70^{\circ} \mathrm{C}$, and high alkali $\mathrm{pH}$ resistance. Moreover, the enzyme is an endo-xylanase [12] and it is free from cellulose activity.

The usage of xylanase enzyme in pulp and paper industry is very minimum due to the limited availability of commercial xylanase enzyme compared to the high demand for paper bleaching process (high heat resistance). So far, xylanse is produced using pure xylene as an inducer which affects the cost of production to be expensive and produces enzyme with exotic characteristic (not inorganic). Enzymes which hasvebeen stored for long without a proper storage method reduces their activity and therefore increase the consumption. Moreover there is a lack of technology transfer in the utilization of enzymes in the industry. The characteristic of xylanase in the market nowadays is having optimum temperature of less than $50^{\circ} \mathrm{C}$ [11] therefore the xylanase available is not in accordance with the industrial needs. There is much effort done in order to improve the thermal stability of the enzyme. One measure that can be used to improve the thermostability of an enzyme is by enzyme engineering.

Temperature stability is one of protein characteristics that are often observed recently since production process, storage to usage are all involving heat. Stability affects protein structure (conformation) which affects the functions. Hydrophobic interaction, salt bridge and the interaction between amino acid with charges like lysine, arginine, aspartic acid, glutamic acid and amino acid from peptide bonds are several important forces that protein structure. Generally, to strengthen those forces for instance, is by altering amino acid to hydrophilic in hydrophobic environment, in order to improve the stability of the protein.

In this study, Aspergillus niger xylanase enzyme Wild Type (AnX) is analyzed for its thermal stability based on hydrophobic interaction through molecular dynamics simulation (MDS).

\section{Materials and Methodology}

\subsection{Materials}

The materials used in this study were experimental data of Diffraction X-Ray which is coordinate data for Aspergillus niger xylanase enzyme [13] which can be downloaded on protein data bank (PDB) (http://www.rscb.org/pdb/) with PDB 1UKR code [14]. 


\subsection{Tools}

The tool is used in this study were hardware and software. The hardware consists of computer with specification such as 8 GB RAM, Quad Core Processor (Intel Core 7), Graphic Card NVIDIA Ge Force GTS 9400, 16 core GPU and LINUX Operations System Ubuntu 14.04 version. The software used for simulation process was NAMD (Not Just Another Molecular Dynamics Program) version 2.9 [15] whereas for preparation and simulation result analysis, VMD (Visual Molecular Dynamics) version 1.9.1 was used [16]. Data processing was done using CatDCD program version 4. VBA Microsoft Excel 2010 was used to smooth graphs and Gnuplot version 2.6 was used to make graphs.

\subsection{Research Design}

\section{Simulation System Preparation}

The crystal structure of Aspergillus niger xylanase enzyme (code PDB: 1 UKR) [7] used in the simulation was obtained from Protein Data Bank (PDB) (http://www.pdb.org/pdb/home/home.do). The crystal structure 1UKR determination was done with X-ray diffraction method with $2.4 \AA$ resolution. Amino acid residues as cylanase constituent were residue 181. Protein Structure File (PSD) is a file with specific molecule information which is needed to apply the certain force field into molecule system. To create this file, NAMD provide psfgen program.

To resemble cellular environment, the protein was dissolved in water. The protein dissolved was in the shape of a box with a size of $100 \AA$ x $100 \AA$ x $100 \AA$ consists of water molecule. Water molecule chosen for explicit solvation in simulation system is TIP3P water molecule model with Periodic Boundary Condition (PBC). This system was neutralized by adding in ion $(\mathrm{NaCl})$ as physiology concentration using VMD solvate and auto ionize plugin.

In this simulation, parameter force field file was needed. A force field is a mathematic expression from potential force obtained in the system. A force field parameter file, CHARMM consists of numeric constants that are needed in order to evaluate force and energy, remembering PSF is a file of structures and atom coordinate. CHARMM parameter can be downloaded from http: //www.charmm.org/. To run the molecular dynamic simulation, a script was needed. A simulation script is a program to run preferred NAMD simulation. NAMD runs configuration file using $\mathrm{Tcl}$ scripting language.

\section{Simulation of Molecular Dynamics}

The simulation was conducted in 4 stages using NAMD program by inserting the initial configuration file. The file acts as system control with the parameters required to run the simulation. The topology file used was par_all27_prot_na_lipid.inp. The first step was minimization which aims to minimize the energy in the molecule. The initial insertion was 
structure and PSF files from molecule preparation. Second was heating. The insertion for this step is the result from minimization. In the beginning of simulation, system temperation was 0 K. Final temperature was varied to $300 \mathrm{~K}, 400 \mathrm{~K}, 450 \mathrm{~K}$ and $500 \mathrm{~K}$. Thirdly, equilibration where the temperature was kept constant using Langevin protocol. Finally, the last step was production run. This is the stage when molecular dynamics simulation ran. The molecule was let to freely move by no longer controlling the temperature in the system. This stage was performed for $10 \mathrm{~ns}$ for $300 \mathrm{~K}, 400 \mathrm{~K}, 450 \mathrm{~K}$ and $500 \mathrm{~K}$.

The output from the last step for molecular dynamics simulation is cdc file. The file was visualized at VMD program. The data obtained produced graphs which are required to analyze protein's thermal stability.

\section{Result and Discussion}

The study of enzyme and protein thermostable has provided new knowledge about the factors which determine enzymes' thermal stability and also the practical practice in industrial field. The understanding of factors determine thermal stability of a protein or an enzyme can be used as a reference for protein engineering also in order to avoid mistakes in target mutation which can lead to fatality of both stability and enzymes' activities.

The research done was divided into 3 groups that include Aspergillus niger xylanase wild-type enzyme thermal stability simulation which is a simulation done to analyze the enzyme's thermal stability, the choosing of Aspergillus niger xylanase enzyme mutant and FEP simulation to analyze the change of free energy of the chosen mutant solvation.

\subsection{Themal Stability Analysis of Aspergilus niger Xylanase Enzyme}

Thermal stability analysis of Aspergillus niger xylanase enzyme can be observed from unfolding process which aims to find out the enzyme's flexibility in the changes of structure occurred. The parameter observed in this analysis was RMSF (root-mean-square fluctuation).

RMSF is the average square root of atom coordinate fluctuations against its reference structure. It is a flexibility analysis of amino acid residues as the constituent of proteins, which can be calculated using the following equation:

$$
\left.\operatorname{RMSF}=\sqrt{\left[\mathrm{R}_{\mathrm{j}}-\left|\mathrm{R}_{\mathrm{j}}\right|\right.}\right]^{2}
$$

where $R_{j}$ is residue's coordinates $j$ and $\left|R_{j}\right|$ represents the average of $j$ residue positions. The analysis was used to get information of the flexible and rigid amino acid residues during the simulation process.

For RMSP parameter in Figure 1.a, 1.b, 1.c and 1.d show that the simulation at $300 \mathrm{~K}, 400 \mathrm{~K}$ and $450 \mathrm{~K}$ did not lead to significant fluctuations of RMSF values. The pattern shown is 
relatively similar at the three temperature variations. This means that the protein is relatively stable despite the peaks occur which captured the flexible residues. However, the amount can be considered as small. Meanwhile at $500 \mathrm{~K}$, RMSF value increased significantly and the pattern produced was far different from the other temperature. With the increased of temperature, the rigid residues become flexible which is marked with the number of peaks occurred. This indicated that unfolding process in the protein has taken place.

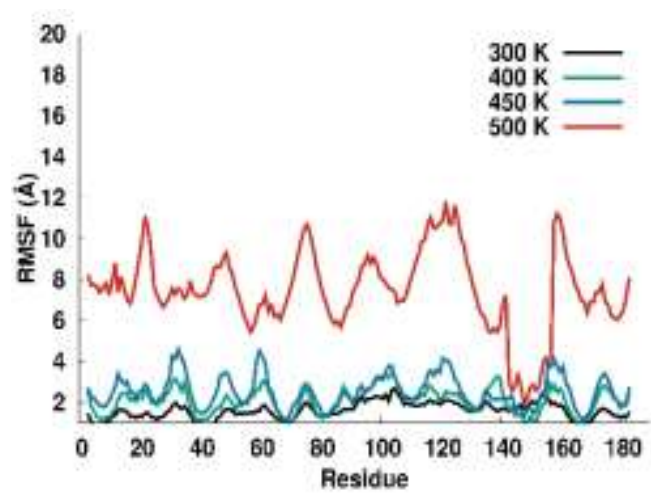

(a)

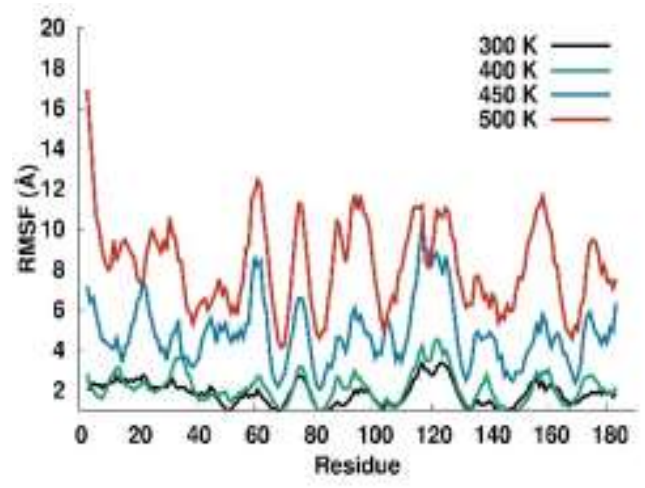

(c)

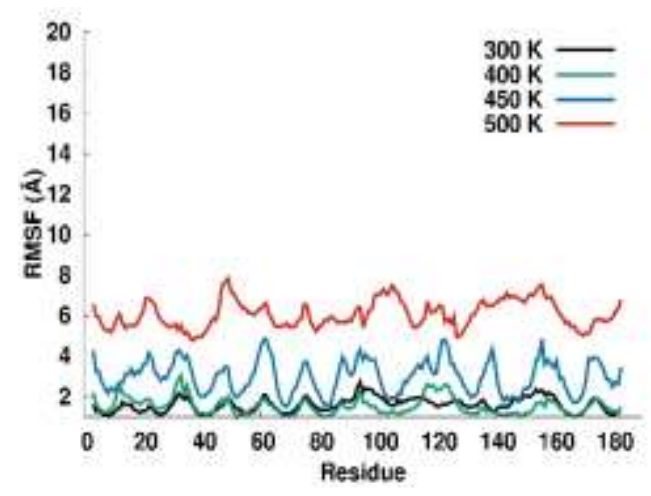

(b)

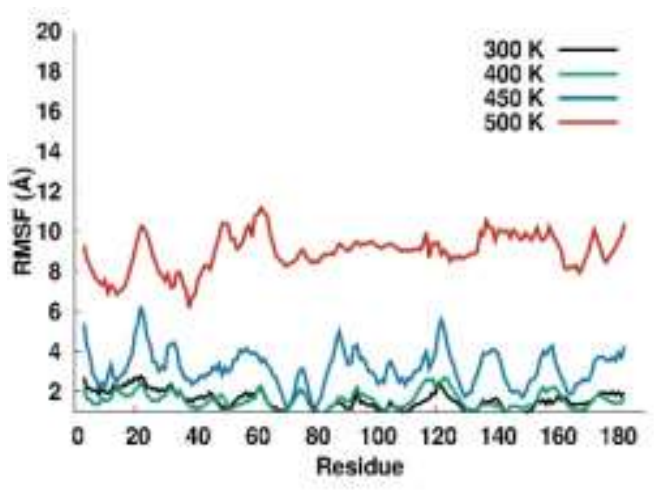

(d)

Figure 1. The changes of enzyme's parameter analysis at $300 \mathrm{~K}, 400 \mathrm{~K}, 450 \mathrm{~K}$ and $500 \mathrm{~K}$ for $10 \mathrm{~ns}$ each (a). RMSF segment 1 (b). RMSF segment 2 (c). RMSF segment 3 (d). RMSF segment 4.

The four segments or chains analyzed show that segment 1 and 3 have unique patterns and significant fluctuation. It was assumed that the residues in chain 1 and 3 have higher fluctuation therefore has the potential to be mutated to make the residue become rigid. 


\subsection{The Selection of Aspergilus niger Xylanase Enzyme}

The selection of residues for mutation to increase Aspergillus niger xylanase enzyme was based on hydrophobic interaction which was considered to be able to determine which residues to posses significant contribution to stabilize the proteins, in this case is the enzyme. The analysis was only done at $500 \mathrm{~K}$ when the protein has undergone unfolding process to ease the findings of residues to stabilize the protein.

Among the factors that determine thermal stability of proteins, hydrophobic interaction was considered as the dominant determinant for protein stability [17]. This is based on the phenomenon that when protein starts folded, most non polar residues $(\sim 80 \%)$ will be buried in proteins' interior, protected it against solvent which causes proteins to be able to maintain its integrity. The phenomenon is also known ashydrophobic effect.

In the RMSF analysis, only the 1st and 3rd segments were observed due to the significant fluctuations occurred in the two segments. It was assumed that the residues in chain 1 and 3 have high fluctuation rate hence the potential to be mutated in order to alter the residue to become rigid. In segmen 1, three flexible residues were obtained. Hydrophobic residue was Pro76 and hydrophilic residues were Ser22 and Thr122. The three residues were located in secondary coil and turn structure therefore they are not suitable to be mutated.

In segmen 3, five flexible residues were obtained such as Ala60, Trp30, Gly158, Pro92 and Tyr75. Hydrophobia residues were Ala60, Trp30, Tyr75 and Pro92 whereas hydrophilic residue was Gly158. The residues at secondary coil and turn structure were Gly158, Tyr75 and Pro92, therefore they are unsuitable to be mutated. Meanwhile, Ala60 and Trp30 residues were are beta-sheet structure in which the structure has bigger damage than alpha-helix's during the unfolding. Therefore, they were considered as broken hydrophobic residues with the increasing temperature applied to the proteins.

The two residues, Ala60 and Trp30, were then mutated and 6 variations of mutant were obtained. They are Ala60Ile, Ala60Met, Ala60Val, Trp30Ile, Trp30Met and Trp30Val. The mutant variations were given to maintain its hydrophobic interactions.

To analyze the effect of mutations against enzyme's thermal stability quantitatively, the change of free energy ( $\Delta \Delta$ Gsolv) was measured using Free Energy Perturbation (FEP) approach. If the value of $\Delta \Delta$ Gsolv is positive, then it indicates that the mutant is far less stable compared to its wild-type and vice versa, negative value of $\Delta \Delta$ Gsolv indicated that the mutant is more stable than the wild-type [18] and [19-20]. 


\subsection{The Analysis of Free Energy of Solavtion}

The output of FEP simulation is $\Delta \mathrm{G}(\mathrm{Kcal} / \mathrm{mol})$ value which is later used as the determination whether the mutation done increase or decrease the stability. $\Delta \Delta$ Gsolv value is obtained from the difference of simulated $\Delta \mathrm{G}$ without solvation (vacuo) and simulation with solvent (aqua) which is defined as $\Delta \Delta \mathrm{Gsolv}(\mathrm{Kcal} / \mathrm{mol})$ or the change of free energy of solvation. The value expresses the total energy required to do mutation. Mutants with negative value of $\Delta \Delta \mathrm{Gsolv}$ are able to improve the stability of protein they simulates and vice versa; positive value mutants decrease the stability of protein they mutated. FEP simulation can be unstable. This condition does not damage protein function therefore it is not advisable to do mutation is the system is unstable.

Table 1 shows the $\Delta \Delta$ Gsolv values of all potential mutants. Mutants Ala60Ile, Ala60Met and Ala60Val were obtained based of hydrophobic interaction analysis. When Free Energy Perturbation (FEP) was simulated, the system became stable. This can be observed from the total energy produced which was headed to negative values range.

Table 1. Mutant Selection and $\Delta \Delta$ Gsolv value

\begin{tabular}{cccc}
\hline Mutant selection analysis & Resiude to be mutated & Mutant & $\begin{array}{c}\Delta \Delta \mathrm{Gsolv} \\
(\mathrm{Kkal} / \mathrm{mol})\end{array}$ \\
\hline \multirow{3}{*}{ Hydrophobic interaction } & Ala 60 & Ile & -14.6198 \\
\cline { 2 - 4 } & & Met & -21.10345 \\
& \multirow{2}{*}{ Trp 30 } & Val & -11.605178 \\
\hline & Met & Unstable \\
& Val & Unstable \\
& & Unstable \\
\hline
\end{tabular}

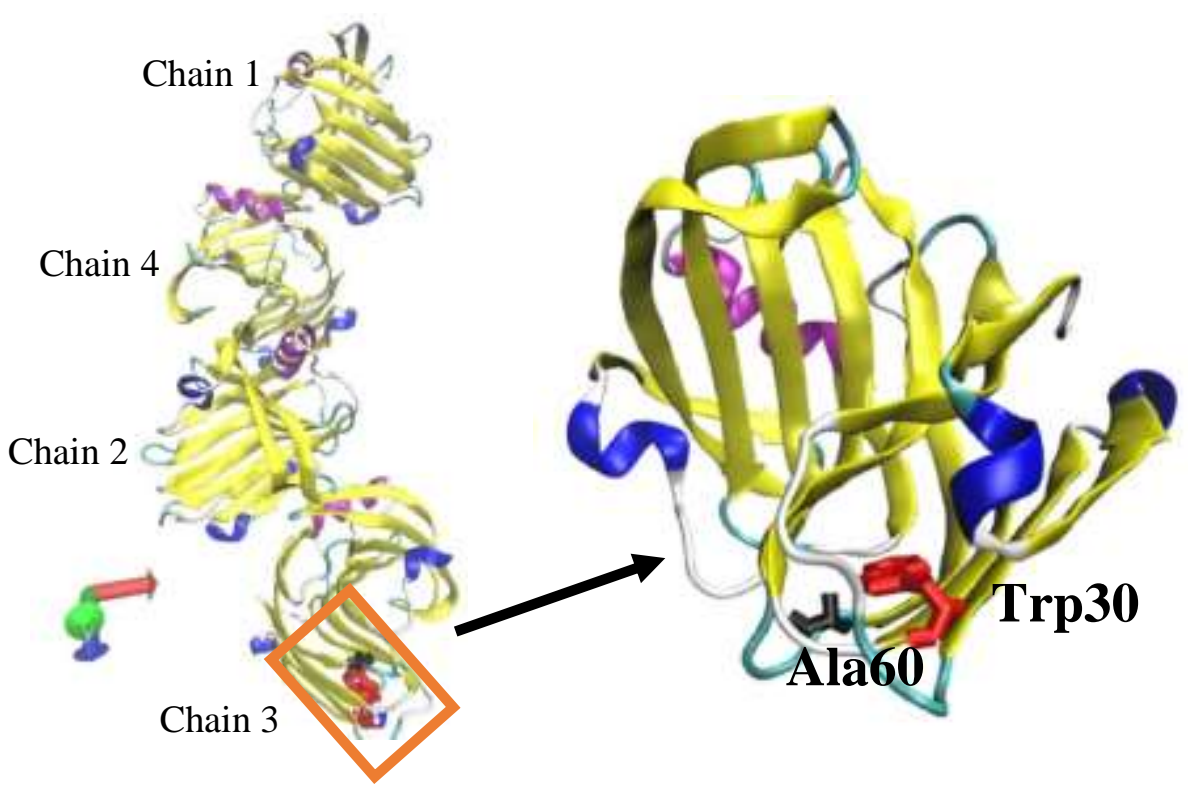

Figure 2. The position of potential mutant based on hydrophobic bonds:

$$
\text { Trp } 30 \text { (red) - Ala } 60 \text { (black) }
$$


If the energy is more towards minimum, then the system is more stable than the wild-type. This means that the mutations done to Ala60Ile, Ala60Met and Ala60Val greatly affect the increase of Aspergillus niger xylanase protein thermal stability. After the mutation, the value of $\Delta \Delta \mathrm{Gsolv}$ for Ala60Ile was negative which means that the system is more stable than the initial condition. The value was -14.6198 while $\Delta \Delta$ Gsolv $=-21.10345$ was obtained by Ala60Met mutant. Negative value of $\Delta \Delta$ Gsolv was also obtained by Ala60Val at -11.605178 . Then from the $\Delta \Delta$ Gsolv values above, it can be concluded that the three mutants of Ala60 are able to improve the stability of Aspergillus niger xylanase protein with the best mutant is Ala60Met since the value is more negative than the other two mutants. It can be said that Ala60Met is the best mutant for Ala60 residue. The three mutations above were aimed to maintain hydrophobic interaction. Only, Ala60 residues went through preference conformation (Biological magnetic resonance data) attached in Attachment 3. The residue has got weak former characteristic in forming $\beta$-sheet conformation with value of 1.67 which makes Metionine residue (60Met) more stable compared to Alanine.

Similarly, mutants of $60 \mathrm{Ile}$ and $60 \mathrm{Val}$ residues have the ability as a strong former $\beta$-sheet with a value of 1.60 and 1.65 respectively therefore Isoleusine (60Ile) and Valine (60Val) residues are more stable than Alanine. However, if we compared the three mutants as a replacement of the strong former $\beta$-sheet, the biggest value obtained was from Metionine residue (60Met). Thues, Ala60Met mutant is the best to improve the stability of Aspergillus niger xylanse protein if the hydrophobic bonds are analysed.

Trp30Ile, Trp30Met and Trp30Va are mutants obtained based on hydrophobic bonds. When FEB simulation was run, the system beame unstable which means that Triptofane (Trp30) is not suitable to be mutated as it causes the system to become unsable. This means that the mutations done to Trp30Ile, Trp30Met and Trp30Val are insignificant to improving thermal stability of Aspergillus niger xylanse protein and it is not advisable to conduct the mutation as protein may broken and loss its functions.

Trp30Ile, Trp30Met and Trp30Va are mutants obtained based on hydrophobic bonds. When FEB simulation was run, the system became unstable which means that Triptofane (Trp30) is not suitable to be mutated as it causes the system to become unstable. This means that the mutations done to Trp30Ile, Trp30Met and Trp30Val are insignificant to improving thermal stability of Aspergillus niger xylanse protein and it is not advisable to conduct the mutation as protein may be broken and loss its functions.

\section{Conclusion}

Aspergillus niger xylanse protein went through unfolding at $500 \mathrm{~K}$ temperature at $9.5 \mathrm{~ns}$ time. The residues accountable for Aspergillus niger xylanse protein thermal stability based on 
hydrophobic interaction is Alanine at residue 60. The two residues are found in segment or chain.

The selection of mutants for Aspergillus niger xylanse protein was based on hydrophobic interaction and the mutant variations obtained were Ala60Ile, Ala60Met, Ala60Val, Trp30Ile, Trp30Met and Trp30Val. The variations were given to maintain the hydrophobic interaction. Free Energy Perturbation (FEP) simulation conducted showed the best and the most possible mutation for Alanine 60 that was replaced by Metionine. This alteration leads to $\Delta \Delta$ Gsolv value of -21.10345. Therefore Ala60Met is the most stable mutant to improve the thermal stability of Aspergillus niger xylanse protein.

\section{Acknowledgement}

The author would like to express the infinite gratitude and thanks to God the Almighty, because the author can finally complete the research. This writing can be done because of the financial aid from Universitas Kristen Indonesia; for that the author would like to say thank you. The author would also like to acknowledge the Dean and Vice Dean of the Faculty of Teacher Training and Education (FKIP) UKI, to the Department Head of Physics Department and to research coordinators of FKIP UKI also the fellow lecturers in FKIP UKI. Thank you for the support, prayers and effort given for the completion of the research.

\section{REFERENCES}

[1] E. A. Falch, "Industrial Enzymes Developments in Production and Application," Biotech Adv., vol. 9, pp. 643-658, 1991.

[2] W. Aehle, Enzymes in Industry: Production and Applications $3^{\text {rd }}$ Edition, Germany: Wiley-VCH Verlag GmbH \& Co. KGaA, 2007.

[3] M. Ghaffari-Moghaddam, H. E. D. Omay and E. Zakipour-Rahimabadi, "Industrial Applications of Enzymes." Review Journal of Chemistry, vol. 4, no. 4, pp. 341-361, 2014.

[4] M. A. Lima, M Oliveira-Neto, M. A. S. Kadowaki, F. R. Rosseto, E. T. Prates, F. M. Squina, A. F. P. Leme, M. S. Skaf and I. Polikarpov, "Aspergillus niger $\beta$-Glucosidase Has a Cellulase-like Tadpole Molecular Shape," The Journal of Biological Chemistry, vol. 288, no. 46, pp. 32991-33005, 2013.

[5] M. L. T. M. Polizeli, A. C. S. Rizzatti, R. Monti, H. F. Terenzi, J. A. Jorge, and D. S. Amorim, "Xylanases from Fungi: Properties and Industrial Applications." Applied Microbiology and Biotechnology, vol. 67, no. 5, pp. 577-591, 2005.

[6] P. Biely, C. R. MacKenzie, J. Puls and H. Schneider, "Cooperativity of Esterases and Xylanases in The Enzymatic Degradation of Acetyl Xylan," Bio/technology, vol. 4, no. 8, pp. 731-733, 1986.

[7] T. K. Kirk and T. W. Jeffries, Roles for Microbial Enzymes in Pulp and Paper Processing, USA: American Chemical Society, 1996.

[8] P. Bajpai, "Application of Enzymes in The Pulp and Paper Industry," Biotechnology Progress, vol. 15, no. 2, pp. 147-157, 1999.

[9] A. Gutiérrez, J. C. D. Rio and A. T. Martínez, "Microbial and Enzymatic Control of Pitch in The Pulp and Paper Industry." Applied Microbiology and Biotechnology, vol. 82, no. 6, pp. 1005-1018, 2009.

[10] Q. Beg, M. Kapoor, L. Mahajan and G. S. Hoondal, "Microbial Xylanases and Their 
Industrial Applications: A Review," Applied Microbiology and Biotechnology, vol. 56, pp. 326-338, 2001.

[11] A. Dhillon, J. K. Gupta and S. Khanna, "Enhanced Production, Purification and Characterisation of A Novel Cellulase-Poor Thermostable, Alkalitolerant Xylanase from Bacillus circulans AB 16," Process Biochemistry, vol. 35, no. 8, pp. 849- 856, 2000.

[12] L. Viikari, A. Kantelinen, J. Sundquist and M. Linko, "Xylanases in Bleaching: from An Idea to The Industry," FEMS Microbiology Reviews, vol. 13, no. 2-3, pp. 335- 350, 1994.

[13] U. Krengel and B. W. Dijkstra, "Three-Dimensional Structure of Endo-1,4-beta-xylanase I from Aspergillus niger: Molecular Basis for Its Low pH Optimum.," J.Mol.Biol., vol. 263, no. 1, pp. 70-78, 1996.

[14] H. M. Berman, J. Westbrook, Z. Feng, G. Gilliland, T. N. Bhat, H. Weissig, I. N. Shindyalov and P. E. Bourne, "The Protein Data Bank," Nucleic Acids Research, vol. 28, no. 1, pp. 235-242, 2000.

[15] J. C. Phillips, R. Braun, W. Wang, J. Gumbart, E. Tajkhorshid, E. Villa, C. Chipot, R. D. Skeel, L. Kale and K. Schulten, "Scalable Molecular Dynamics with NAMD," J. Comput. Chem., vol. 26, no. 16, pp. 1781-1802, 2005.

[16] W. Humphrey, A. Dalke and K. Schulten, "VMD: Visual Molecular Dynamics," J. Mol. Graph., vol. 14, no. 1, pp. 33 - 38, 1996.

[17] C. K. Mathews and K. E. Van Holde, Biochemistry. 2nd., United States: The Benjamin/Cummings Publishing Company, Inc., 1996.

[18] P. Kollman, "Free Energy Calculations: Applications to Chemical and Biochemical Phenomena," Chem Rev., vol. 93, no. 7, pp. 2395-2417, 1993.

[19] A. Ghoufi, C. Bonal, J. P. Morel, N. Morel-Desrosiers and P. Malfreyt, "Gibbs Free Energy Perturbation Calculations, An Application to the Binding of Alkylammonium Cations by Water-Soluble Calixarene," J. Phys. Chem. B., vol. 108, no. 31, pp. 11744-11752, 2004.

[20] B. G. Ra, and U. C. Singh, "Hydrophobic Hydration: A Free Energy Perturbation Study," Journal of the American Chemical Society, vol. 111, no. 9, pp. 3125-3133, 1989. 